\title{
Avances en la prevención de eventos tromboembólicos en pacientes con fibrilación auricular
}

\author{
Rodrigo Montagna $\mathrm{M}^{1,2}$, René Asenjo $\mathrm{G}^{1,2}$, \\ Raimundo Morris $\mathrm{C}^{1}$, Mario 0 rtiz $0^{1}$, Mauricio Cereceda $\mathrm{B}^{1}$. \\ Update on the prevention of \\ thromboembolic phenomena \\ in atrial fibrillation
}

Atrial fibrillation is the most common sustained arrhythmia in clinical practice and is associated to thromboembolic complications. Anticoagulation with vitamin $\mathrm{K}$ antagonists is clearly useful to reduce the incidence of emboli, but associated with important limitations. Therefore, there is an active search for medications that are more effective and simpler to prescribe and manage. Synthetic pentasaccharides of heparin such as idraparinux for parenteral use, showed promising results. Direct inhibitors of thrombin were also useful for the prevention of thromboembolism. However, they were withdrawn from the market due to potentially fatal adverse reactions. Other area of investigation has been the effectiveness of the combination of antiplatelet agents such as aspirin and clopidrogel. Although this combination is attractive, results of clinical trials must be awaited to have an opinion about its real usefulness. Finally, left atrial appendage transcatheter occlusion (PLAATO) is an effective and reasonably safe method for patients with contraindications for anticoagulation or those that continue to embolize despite well prescribed anticoagulation. The long term results of this intervention must also be awaited (Rev Méd Chile 2007; 135: 1048-55).

(Key w ords: Anticoagulants; Atrial fibrillation; Thromboembolism)

Recibido el 8 de septiembre, 2006. Aceptado el 20 de noviembre, 2006.

${ }^{1}$ Unidad de Arritmias, Centro Cardiovascular, Hospital Clínico de la Universidad de Chile.

2Departamento de Cardiología, Clínica Alemana de Santiago, Chile.

L

a fibrilación auricular (FA) es el trastorno del ritmo sostenido más frecuente en la práctica clínica, condicionando una mayor morbilidad y mortalidad en quien la padece ${ }^{1}$. Sabemos actualmente que la FA induce alteraciones estructurales

Correspondencia a: Dr. Rodrigo Montagna M. Hospital Clínico de la Universidad de Chile. Centro Cardiovascular. Santos Dumont 999, 3o piso, sector E. Santiago. Fono: 7378038. Fax: 7320683. E mail: rmontagna900@msn.com y funcionales en el miocardio auricular y ventricular y si se mantiene por tiempo prolongado, particularmente si la respuesta ventricular es rápida, llevará a la dilatación de la aurícula y ventrículo izquierdo, disminución de la fracción de eyección y desarrollo de insuficiencia cardíaca, fenómeno conocido como taquicardiomiopatía ${ }^{1,2}$.

Por otra parte, es bien conocida su relación con el desarrollo de complicaciones tromboembólicas (TE), las que pueden tener consecuencias invalidantes o conducir a la muerte ${ }^{3}$. En años recientes hemos 
conocido los resultados de importantes ensayos clínicos controlados (ECC) sobre el control de ritmo y control de frecuencia asociado a terapia anticoagulante (TAC) en pacientes con $\mathrm{FA}^{4}$. Aun cuando el debate continúa, la gran enseñanza de estos ECC ha sido lo esencial que resulta el uso del TAC, tanto en los pacientes en que se privilegia el control de la frecuencia, como en aquellos en que se opta por una estrategia del control del ritmo, pues en este último grupo la tasa de recurrencia de la FA en el mediano y largo plazo es alta, del orden de 30\%$50 \%$, y en un porcentaje importante de los pacientes esta recaída es asintomática u oligosintomática, exponiéndolos a complicaciones embólicas ${ }^{5}$.

A pesar de la bien establecida utilidad de la anticoagulación en la $\mathrm{FA}^{6}$, esta terapia presenta varias limitaciones, lo que ha determinado una activa investigación en esta área, tanto en la búsqueda de mejores fármacos anticoagulantes como en novedosas formas de prevención de embolias.

En 1998 revisamos la terapia anticoagulante para prevenir embolias en pacientes con $\mathrm{FA}^{7}$. En el presente artículo analizaremos los avances más recientes en esta terapia, discutiendo nuevos fármacos y técnicas no farmacológicas.

Limitaciones de los anticoagulantes orales. Los anticoagulantes más ampliamente utilizados en el mundo son los antagonistas de la vitamina $\mathrm{K}$ (AVK). Para que su efecto llegue a ser mensurable deben disminuir los niveles funcionales de los factores dependientes de esta vitamina, lo que lleva habitualmente 3 a 5 días, determinando un inicio de acción lento, por lo que con frecuencia deben superponerse con heparinas en las etapas iniciales del tratamiento. La respuesta a las dosis es variable por lo que se hace imprescindible medir su efecto a través del INR en forma permanente ${ }^{8}$. Lo anterior supone un inconveniente para pacientes y médicos y costos importantes para el sistema de salud ${ }^{8}$.

Los ECC de la década 1990-99 definieron claramente la intensidad de la anticoagulación para prevenir embolias y minimizar las complicaciones hemorrágicas. Sabemos actualmente que la mayoría de los pacientes requieren mantener un nivel de anticoagulación con INR entre 2,0 y 3,0, lo que implica un margen terapeútico muy estrecho $0^{6,9-11}$. Derivadas de las limitaciones farmacológicas pre- viamente enunciadas, se desprenden muchas de las limitaciones clínicas del TAC. Es conocido que los pacientes incluidos en los ECC, en ésta y otras áreas, no representan necesariamente al gran número de pacientes con FA de la práctica clínica habitual. En los ECC existen una gran cantidad de criterios de exclusión para someter a pacientes a TAC y al mismo tiempo el control del mismo se efectúa en forma muy rigurosa, lo que hace que sus resultados no sean completamente extrapolables a la población general ${ }^{12,13}$.

Lo anterior podría explicar, en parte, las múltiples experiencias en hospitales con población no seleccionada que evidencian que de los pacientes elegibles para $\mathrm{TAC}$, según las guías clínicas actuales, sólo un porcentaje bajo está recibiendo anticoagulación ${ }^{14-19}$.

Por otro lado, el TAC supone un riesgo de sangrado evidente y bastante bien definido ${ }^{9,20}$. La incidencia de sangrado mayor asociado a warfarina es significativamente más elevada que placebo, 2,2\% versus $0,9 \%$ pacientes/año, y que antiplaquetarios como el ácido acetil salicílico (ASA): 1,6\% vs 1,0\% pacientes/año. En forma similar, la tasa de hemorragia intracraneal (HIC) fue el doble en aquellos en TAC en relación al placebo: $0,4 \%$ vs $0,2 \%$ pacientes/ año, y fue mayor que con el uso de ASA: 1,2\% versus $0,5 \%$ pacientes/año ${ }^{9,20}$. La incidencia de sangrado está directamente relacionada con el nivel de INR, aumentando en forma evidente cuando éste es mayor de $3^{20}$. Cabe destacar que la gran mayoría de las investigaciones en FA han sido efectuadas con warfarina, y en nuestro país hemos extrapolado esos resultados al acenocumarol, anticoagulante con algunas diferencias farmacológicas respecto de la warfarina, y que aún es el más usado en nuestro medio. Aunque no conocemos estudios comparativos en esta área, nuestra impresión es que si se obtiene un INR adecuado los resultados y complicaciones son similares a los descritos con warfarina.

\section{NuEVAS ESTRATEgias}

\section{A) Farmacológicas}

1) Idraparinux. Se trata de un análogo sintético de la secuencia pentasacárida de la heparina. A diferencia de las heparinas que provienen de fuentes animales, el idraparinux es producido por 
síntesis química. La molécula se une en forma selectiva e irreversible a la antitrombina, lo que induce un cambio conformacional en esta molécula determinando un aumento de afinidad por el factor $\mathrm{X}$ activado (FXa) en más de 300 veces. Este complejo irreversible antitrombina-FXa inhibe la acción del $\mathrm{FXa}$, lo que resulta en la inhibición de la formación de trombina. A diferencia de la heparina y heparinas de bajo peso molecular (HBPM) no se evidencia unión del idraparinux al factor plaquetario $4 \mathrm{u}$ otras proteínas del plasma, lo que contribuye a obtener un efecto farmacológico muy predecible y, por lo tanto, no requiere monitorización de su efecto ${ }^{21}$.

Debe ser administrado en forma parenteral y, al tener una vida media plasmática cercana a las $80 \mathrm{~h}$ luego de la administración subcutánea, puede dosificarse una vez a la semana ${ }^{21}$. Aun cuando la administración parenteral puede ser una desventaja, una inyección subcutánea semanal pudiera ser preferible, para muchos pacientes, que el control permanente del INR. Una limitación adicional de este nuevo fármaco es la ausencia de antídoto. A diferencia de la heparina y las HBPM en menor grado, el sulfato de protamina no revierte el efecto anticoagulante del idraparinux, lo que, sumado a su larga vida media, puede ser problemático en el momento de requerir reversión urgente de su acción. Se ha mostrado útil la administración de factor VIIa recombinante como agente neutralizador de los pentasacáridos sintéticos, pero su disponibilidad en nuestro medio es restringida 22,23 .

Se encuentra en desarrollo el ECC AMADEUS, estudio abierto de fase III que comparará en más de 7.000 pacientes con FA los efectos del idraparinux (administrado en forma subcutánea una vez por semana sin control de la anticoagulación) con TAC oral a dosis ajustadas para lograr un INR entre 2,0 y 3,0 .

2) Inhibidores directos de la trombina (IDT):

a) IDT parenterales: A diferencia de la heparina no fraccionada y las HBPM que ejercen su acción sobre la trombina, en forma indirecta, este grupo de fármacos se une a sitios específicos de la trombina ejerciendo una acción directa sobre esta molécula ${ }^{24}$.

Se encuentran disponibles para uso clínico tres IDT de administración parenteral: hirudin, bi- valirudin y argatroban. Ninguno ha sido evaluado en prevención de complicaciones TE en pacientes con FA, sin embargo, han sido extensamente estudiados en otras áreas, considerando sus potenciales ventajas sobre las heparinas: pacientes con síndromes coronarios agudos, trombopenia inducida por heparina, profilaxis y tratamiento del tromboembolismo venoso. En esas poblaciones, su eficacia fue al menos equivalente a la heparina y con perfiles de seguridad aceptables. Algunos centros están utilizando estos fármacos en pacientes sometidos a angioplastia coronaria ${ }^{25,26}$.

b) IDT orales: Ximelagatrán: Es una prodroga que requiere su transformación en melagatrán, el principio activo. Luego de la administración oral, el ximelagatrán es absorbido desde el tubo digestivo con una biodisponibilidad cercana a 20\%, alcanzando una concentración plasmática máxima a los $30 \mathrm{~min}$. Luego de su transformación en melagatrán, éste se une al sitio activo de la trombina bloqueando la actividad catalítica de la enzima. Tiene una vida media de $4-5 \mathrm{~h}$ y debe, por lo tanto, administrarse dos veces al día.

El melagatrán es eliminado principalmente por vía renal, prolongándose su vida media en pacientes con insuficiencia renal.

No requiere ajuste de dosis en relación a la edad, sexo, raza ni tipo de dieta, lo que implica que puede indicarse en dosis fijas en la mayoría de los pacientes ${ }^{27}$. El ximelagatrán ha sido estudiado en diversas condiciones para las cuales la anticoagulación con AVK es considerada actualmente el estándar, como en prevención y tratamiento del TE venoso, posterior a la fase aguda de un infarto agudo al miocardio (IAM) y en pacientes con $\mathrm{FA}^{27,28}$.

Dos ECC de reciente publicación en pacientes con FA han evaluado la eficacia del ximelagatrán administrado sin monitorización de la coagulación, en comparación con dosis ajustadas de warfarina para mantener el INR entre 2,0 y 3,0. Ambos estudios fueron diseñados como ensayos de no inferioridad»con miras a demostrar que el ximelagatrán es al menos tan efectivo y tolerado como la warfarina. El primero de estos ECC, el SPORTIF III ${ }^{29}$, que fue un estudio abierto, tuvo como punto final de eficacia la incidencia combinada de cualquier accidente vascular encefálico 
(ACV) (isquémico o hemorrágico) y eventos embólicos sistémicos. El punto final de seguridad fue la incidencia de complicaciones hemorrágicas mayores y menores. Después de un seguimiento promedio de 17 meses, 2,2\% de los pacientes asignados a warfarina presentaron alguna complicación embólica, versus 1,8\% del grupo con ximelagatrán, diferencia que fue significativa. No existieron diferencias significativas en relación a la incidencia de complicaciones hemorrágicas mayores: $1,8 \%$ versus $1,3 \%$ en los pacientes con warfarina y ximelagatrán, respectivamente.

Como reacción adversa adicional, se ha comunicado un aumento de la alanino amino transferasa (ALT) sobre tres veces el límite de lo normal en $14(1 \%)$ de los pacientes en el grupo con warfarina y en 107 (6\%) de los asignados a ximelagatrán $(p<0,0001)^{29}$. En este último grupo, el aumento de la ALT ocurrió típicamente entre los 2 y 6 meses después del inicio del tratamiento y regresó a niveles normales, sin consecuencias clínicas, ya sea espontáneamente (59 pacientes) o luego de la interrupción del ximelagatrán (48 pacientes) ${ }^{29}$.

$\mathrm{Al}$ estudio SPORTIF III le siguió el ECC, esta vez en forma aleatoria y doble ciego, SPORTIF V ${ }^{30}$. En este estudio, que incluyó 3.922 pacientes de 409 centros norteamericanos, los eventos finales a evaluar fueron los mismos que en el SPORTIF III. Luego de un seguimiento de 6.405 pacientes/ año (promedio 20 meses) la tasa de incidencia de ACV o embolias sistémicas en los pacientes asignados a ximelagatrán fue de 1,6\% por año y de $1,2 \%$ por año en aquellos que recibieron warfarina, diferencia no significativa. Tampoco existió una diferencia estadísticamente significativa en la incidencia de complicaciones hemorrágicas mayores, $2,4 \%$ de pacientes en el grupo del ximelagatrán y 3,1\% pacientes en el grupo con warfarina $^{30}$. Como en el SPORTIF III, debe considerarse que los pacientes que recibieron warfarina tuvieron un muy buen control de la anticoagulación, con INR dentro del rango sextendido » de 1,8 a 3,2 en 83\% de las mediciones durante todo el seguimiento. Al igual que en el SPORTIF III, los pacientes que recibieron ximelagatrán presentaron mayor incidencia de elevación de la ALT (sobre 3 veces el límite mayor de la normalidad) que aquellos en terapia con warfarina. En los primeros, se registró elevación de ALT en 6\% del grupo, usualmente en los primeros 6 meses y típicamente esta alteración se normalizó independientemente de la continuación del ximelagatrán. Sin embargo, se comunicó un caso de falla hepática que terminó en muerte y otro caso sugerente de hepatotoxicidad severa. El desarrollo de hepatotoxicidad potencialmente grave condicionó el rechazo por parte de la Food and Drug Administration de los Estados Unidos de Norteamérica (FDA) para la comercialización de este fármaco en ese país $\mathrm{y}$, recientemente, la casa farmacéutica dueña de la patente anunció el retiro del ximelagatrán del mercado.

A pesar de lo anterior, es posible que la investigación con esta familia de fármacos prosiga, intentando mantener el excelente perfil de efectividad y superando las reacciones adversas comentadas.

3) Antiplaquetarios. Aspirina más clopidogrel: Estos agentes antiplaquetarios poseen diferentes mecanismos de acción, lo que determina un mayor efecto sobre la agregación plaquetaria al usarse en forma combinada que cada uno en forma aislada. Las primeras evidencias de la utilidad de la combinación de 2 antiplaquetarios en la prevención de TE en pacientes con FA proviene del estudio ESPS $\mathrm{II}^{31}$. Este ECC evaluó la efectividad y perfil de riesgo de una formulación de acción prolongada de dipiridamol, utilizado como agente único o en combinación con ASA en pacientes que habían presentado un ACV isquémico o un accidente isquémico transitorio (AIT). La terapia de combinación resultó ser más efectiva que el uso de cualquiera de los agentes en forma aislada. En el subgrupo de pacientes con FA también existió una tendencia hacia la superionidad del tratamiento combinado. A diferencia del anterior, el estudio MATCH, que comparó el uso de clopidogrel con la combinación de clopidogrel con ASA en pacientes con ACV reciente o AIT y al menos un factor adicional de riesgo vascular no mostró diferencias significativas en la incidencia combinada de ACV isquémico, IAM, muerte de origen vascular 0 rehospitalización por complicaciones cerebrovasculares $^{32}$. La incidencia de hemorragias graves fue significativamente mayor en los pacientes con ASA y clopidogrel que en aquellos en terapia sólo con clopidogrel: 2,6\% versus 1,3\%. Debe considerarse que este ECC no fue diseñado para evaluar el 
efecto de estas terapias en pacientes con FA. De hecho, aunque en el ECC no se menciona directamente el número de pacientes en $\mathrm{FA}, 3 \%$ de los pacientes presentaron un ACV cardioembólico ${ }^{32}$.

El estudio ACTIVE, ECC multicéntrico, diseñado para evaluar la utilidad de la terapia de combinación ASA/clopidogrel versus warfarina en dosis habituales, se encuentra actualmente en pleno desarrollo. Este ECC comprende dos estudios separados, conocidos como ACTIVE-W y ACTIVE-A. La rama ACTIVE-A evaluará la terapia de combinación ASA/clopidogrel versus ASA como agente único en pacientes con contraindicación para warfarina o que rechacen el TAC oral. En pacientes elegibles para terapia anticoagulante, el estudio ACTIVE-W compararía la combinación de ASA y clopidogrel versus dosis ajustadas de warfarina para obtener INR de 2,533. El ACTIVE tiene un diseño factorial $2 \times 2$, de forma tal que los pacientes incluidos en las ramas ACTIVE-A y ACTIVE-W además reciben en forma aleatoria irbesartán o placebo, para investigar los efectos de este fármaco en la disminución de los eventos vasculares. Recientemente se ha comunicado la detención, antes de lo programado, de la rama ACTIVE-W por recomendación del comité interno de seguridad, al comprobarse una superioridad de la warfarina sobre ASA/clopidogrel en la prevención de eventos vasculares. Así, las evidencias muestran que la combinación ASA/clopidrogel no da suficiente seguridad en pacientes con FA, pero aún existe la posibilidad que esta combinación pueda beneficiar a aquellos pacientes que no son candidatos a warfarina.

4) Combinación de AVK y antiplaquetarios. El uso combinado de ASA con AVK en dosis bajas y fijas no es algo nuevo. La posibilidad teórica que al asociar ASA a warfarina en minidosis, sin necesidad de controlar el INR, pudiera mantener la eficacia anticoagulante y disminuir los riesgos de hemorragia, fue evaluada en el ECC SPAF III, publicado en $1996^{34}$.

Estos investigadores encontraron un riesgo de ACV isquémico 4 veces mayor en los pacientes con FA tratados con warfarina en dosis bajas y fijas (INR promedio 1,3) asociada a ASA (325 mg diarios) en relación a aquellos tratados con warfarina en dosis ajustadas. El riesgo de hemonagias tampoco fue menor en aquellos asignados a la terapia de combi- nación ${ }^{34}$. De esta forma, la combinación de AVK en dosis fijas y bajas con ASA pareció ser una estrategia sin futuro. Sin embargo, recientemente se han comunicado los resultados del ECC NASPEAF, estudio multicéntrico español que evaluó el uso de trifusal, un inhibidor de la cicloxigenasa relacionado a ASA, con eficacia similary menor incidencia de hemorragias, en combinación con warfarina en dosis bajas versus warfarina con INR objetivo de 2,0 a 3,0 ${ }^{35}$. Después de un seguimiento de 2,76 años, la incidencia combinada de muerte de causa vascular y ACV no fatal o embolismo sistémico, fue menor en los pacientes con terapia combinada, y no hubo diferencias en la tasa de complicaciones hemorrágicas ${ }^{35}$.

Aún no se encuentra completamente definido el lugar que podría ocupar la terapia de combinación de warfarina con antiplaquetarios, especialmente con ASA, de uso mucho más difundido que el trifusal.

\section{B) No farmacológicas}

Un porcentaje importante de pacientes con FA tienen contraindicaciones para el TAC, o éste es inefectivo por aparición de complicaciones TE a pesar de un régimen terapeútico bien controlado. Conociendo que en más de $90 \%$ de los eventos embólicos la fuente de origen es la orejuela de la aurícula izquierda $(\mathrm{OI})^{36}$, se ha concebido su exclusión mediante técnicas de acceso pencutáneo. Desde hace décadas, esta aproximación se ha efectuado mediante cirugía convencional en pacientes que van a reemplazo valvular mitral y, más recientemente, se han comunicado resultados similares con una técnica de exclusión de OI mediante videotoracoscopia ${ }^{37}$. Sin embargo, con estas técnicas quirúrgicas existe la posibilidad de que la exclusión sea incompleta, lo que ocurriría aproximadamente en $33 \%$ de los casos en el posoperatorio precoz y en $44 \%$ en el seguimiento alejado mediante ecocardiografía transesofágica (ETE) ${ }^{38}$.

En 2002, se comunicó la primera experiencia con la exclusión de la OI mediante técnica percutánea y desde entonces diversos grupos han dado a conocer sus resultados con este novedoso abordaje ${ }^{39-41}$.

La técnica consiste en ocluir la OI mediante un dispositivo autoexpandible en forma de canastillo y recubierto con politetrafluoroetileno (PTFE), para lo cual se requiere acceder a la AI mediante punción transeptal. El sistema se ha denominado PLAATO 
(percutaneous left atrial appendage transcatheter occlusion) y la liberación del mismo en la OI se realiza mediante guía angiográfica o ETE. La mayor expeniencia comunicada a la fecha proviene de un estudio multicéntrico realizado en hospitales europeos, de Canadá y Estados Unidos de Norteamérica ${ }^{42}$. En esta experiencia, 111 pacientes con FA, contraindicación a TAC y al menos un factor de riesgo adicional para TE, fueron sometidos al PLAATO, siendo efectiva en 109 (97,3\%). Un paciente requirió cirugía de urgencia por taponamiento cardíaco no controlado por medios percutáneos y finalmente presentó muerte cerebral. Otros 3 pacientes requirieron pericardiocentesis debido a hemopericardio. En el seguimiento a 9,8 meses, 2 pacientes presentaron ACV isquémico. No se observó migración del dispositivo ni desamollo de trombos móviles durante el seguimiento con ETE efectuado al mes y 6 meses posterior al implante. Se concluyó que la oclusión de la OI con el sistema PLAATO es factible y con un riesgo aceptable, pudiendo constituir una alternativa en pacientes con FA y contraindicación para el TAC ${ }^{42}$.

Estos interesantes resultados deberán ser confirmados en el seguimiento a largo plazo, especialmente la seguridad del dispositivo y la reducción de complicaciones TE.

\section{ConCLUSIÓN}

La anticoagulación es parte fundamental en el manejo de pacientes con FA permanente o paroxística. La warfarina tiene un papel bien ganado por su eficacia y buen perfil de seguridad cuando se usa en forma adecuada. Los promisorios reportes con ximelagatrán, con resultados clínicos

\section{REFERENCIAS}

1. Greenlee R, Vidailet $H$. Recent progress in the epidemiology of atrial fibrillation. Current Opinion in Cardiology 2005; 20: 7-14.

2. Vidailet H, Granada JC, Chyou PH. A populationbased study of mortality among patients with atrial fibrillation and flutter. Am J Med 2002; 113: 365-70.

3. Atrial FibriLation Investigators. Risk factors for stroke and efficacy of antithrombotic therapy in atrial fibrillation. Analysis of pooled data from comparables a la warfarina y la ventaja de no requerir control de INR, se esfumaron al conocerse la incidencia de complicaciones hepáticas que determinaron su retiro del mercado. Sin embargo, es esperable que la investigación prosiga con esta nueva familia de medicamentos. El uso de combinaciones de antiplaquetarios ha sido también explorado, y recientemente se demostró que ASA más clopidrogel tiene una eficacia menor que la warfarina, pero aún está pendiente cuál es el rol de esa combinación en pacientes que no pueden recibir warfarina. Otro esquema investigado es la asociación de un antiplaquetario con dosis bajas de un AVK. La reciente comunicación del uso de trifusal (antiplaquetario relacionado a la ASA) más warfarina, con resultados similares a los obtenidos con warfarina en dosis optimizada, es muy alentadora, pero requiere más estudios.

Sin duda que el uso de idraparinux una vez por semana, y sin control de INR, parece muy atractivo, pero su perfil de eficacia y seguridad lo conoceremos con los estudios en marcha.

De esta forma, pese a los avances comentados, aún no disponemos de un fármaco que haya logrado equiparar la eficacia de los AVK y, al mismo tiempo, superar las muchas limitaciones que éstos poseen y que determinan, al menos en parte, la subutilización del TAC oral en pacientes con FA a pesar de la contundente evidencia de su beneficio en este grupo de pacientes.

Finalmente, la disponibilidad de un dispositivo para excluir percutáneamente la orejuela izquierda, principal fuente de embolias, es una alternativa terapéutica a considerar en aquellos pacientes que han embolizado y no pueden recibir TAC, o que embolizan a pesar de un tratamiento bien llevado.

five randomized controlled trials. Arch Intern Med 1994; 154: 1449-57.

4. The Atrial Fibrilation Follow-Up Investigation of Rhythm Management (AFFIRM) Investigators. A comparison of rate control and rhythm control in patients with atrial fibrillation. N Engl J Med 2002; 347: 1825-33.

5. Nichol G, Mcalster F, Pham B, Laupacis A, Shea B, GrEen M ET AL. Meta-analysis of randomised controlled trials of the effectiveness of antiarrhythmic agents at promoting sinus rhythm in patients with atrial fibrillation. Heart 2002; 87: 535-43. 
6. Hart R, Benavente O, McBride R. Antithrombotic therapy to prevent stroke in patientes with atrial fibrillation: a meta-analysis. Ann Int Med 1999; 131: 492-501.

7. Montagna R, Asenjo R, Madariaga R. Tratamiento anticoagulante en fibrilación auricular: aún no está todo resuelto. Rev Méd Chile 1998; 126: 689701.

8. Hirsh J, Dalen J, Anderson DR. Oral anticoagulants: mechanism of action, clinical efectiveness, and optimun therapeutic range. Chest 2001; 119 (Suppl.1): 85-21S.

9. Hylek E, Skates S, Sheehan M, Singer D. An analysis of the lowest effective intensity of prophylactic anticoagulation for patients with nonrheumatic atrial fibrillation. N Engl J Med 1996; 335: 540-6.

10. Sherman D, Kim S, Boop B, Corley S, Dimarco J, HART R ET AL. For the National Heart, Lung, and Blood Institute AFFIRM Investigators. Occurrence and Characteristics of Stroke Events in the Atrial FibRiLation Folow-up Investigation of Sinus Rhythm Management (AFFIRM) Study. Arch Intern Med 2005; 165: 1185-91.

11. Hyiek E, Go A, Chang Y, Jensvold N, Henault L, SeLBY J ET AL. Effect of Intensity of Oral Anticoagulation on Stroke Severity and Mortality in Atrial Fibrillation. N Engl J Med 2003; 349: 1019-26.

12. TonkIn A. Evaluation of large scale clinical trials and their application to usual practice. Heart 2000; 84: 679-84.

13. Emmerich J, Le Heuzey JY, Bath P, Connowy S. Indication for antithrombotic therapy for atrial fibrillation: reconciling the guidelines with clinical practice. Eur Heart J 2005; 7(Suppl C): C28-C33.

14. Tapson V, Hyers T, Waldo A, Balard D, Becker R, CAPRINI J ET aL. For the NABOR (National Anticoagulation Benchmark and Outcomes Report) Steering Committee. Antithrombotic Therapy Practices in US Hospitals in an Era of Practice Guidelines. Arch Intern Med 2005; 165: 1458-64.

15. StaFFord R, Radiey $D$. The underutilization of Cardiac Medications of Proven Benefit, 1990 to 2002. J Am Coll Cardiol 2003; 41: 56-61.

16. Pérez I, Melbourn A, Kalra L. Use of antithrombotic measures for stroke prevention in atrial fibrillation. Heart 1999; 82: 570-4.

17. White R, McBurnie M, Manolio T. Oral Anticoagulation in patients with atrial fibrillation: Adherence with guidelines in an elderly cohort. Am J Med 1999; 106: 165-71.
18. Deplanque D, Leys D, Parnetti L, Schmidt R, Ferro J, De Reuck J Et AL. The SAFE II Investigators. Stroke prevention and atrial fibrillation: reasons leading to an inappropriate management. Main results of the SAFE II study. Br J Clin Pharmacol 2004; 57: 798-806.

19. Peterson G, Boom K, Jackson S, Vial J. Doctors' beliefs on the use of antithrombotic therapy in atrial fibrillation: identifying barriers to stroke prevention. Intern Med J 2002; 32: 15-23.

20. Segal J, McNamara R, Miuer M. Anticoagulants or antiplatelet therapy for non-rheumatic atrial fibrillation and flutter. Cochrane Database Syst Rev 2001; 1: CD001938.

21. Samama M, Gerotziafas G. Evaluation of the pharmacological properties and clinical results of the synthetic pentasaccharide (fondaparinux). Thrombosis Research 2003; 109: 1-11.

22. Bijsterveld N, Moons A, Boekholdt M, Van Aken B, FenNema H, Peters R et al. Ability of recombinant factor VIIa to reverse the anticoagulant Effect of the Pentasaccharide Fondaparinuxin Healthy Volunteers. Circulation 2002; 106: 2550-4.

23. Bijsterveld N, Vink R, Van Aken B, Fennema $\mathrm{H}$, Peters R, MejJers J et al. Recombinant factor VIIa reverses the anticoagulant effect of the longacting pentasaccharide idraparinux in healthy volunteers. Br J Haematol 2004; 124: 653-8.

24. BECKER R. Understanding the dynamics of thrombin in cardiovascular disease: Pathobiology and biochemistry for the clinician. Am Heart J 2005; 149 (1S): S2-S8.

25. Gurm H, BhatT D. Thrombin, an ideal target for pharmacological inhibition: A review of direct thrombin inhibitors. Am Heart J 2005; 149: S43-53.

26. Whiте C. Thrombin-directed inhibitors: Pharmacology and clinical use. Am Heart J 2005; 149: S54-60.

27. GuSTAFSSON D. Oral direct thrombin inhibitors in clinical development. Journal of Internal Medicine 2003; 254: 322-34.

28. WaLientin L, Wilcox RG, Weaver WD. Oral ximelagatran for secondary prophylaxis after myocardial infarction: the ESTEEM randomized controlled trial. Lancet 2003; 362: 789-97.

29. OLsson SB. Stroke prevention with the oral direct thrombin inhibitor ximelagatran compared with warfarin in patients with non-valvular atrial fibrillation (SPORTIF III): randomised controlled trial. Lancet 2003; 362: 1691-8. 
30. Sportif Executive Steering Committee For The SporTIF V InVeSTigators. Ximelagatran vs Warfarin for Stroke Prevention in Patients With Nonvalvular Atrial Fibrillation A Randomized Trial. JAMA 2005; 293: 690-8.

31. Diener H, Forbes C, Riekrinen P. European Stroke Prevention Study 2: efficacy and safety data. J Neurol Sci 1997; 151(Suppl 1): S1-61.

32. Diener H, Bogousslavsky J, Brass L, Cimminiello C, Csiba L, Kaste M et al. On behalf of the MATCH investigators. Aspirin and clopidogrel compared with clopidogrel alone after recent ischaemic stroke or transient ischaemic attack in high-risk patients (MATCH): randomized, double-blind, placebo-controlled trial. Lancet 2004; 364: 331-7.

33. Hohnloser S, ConNoLy S. Combined antiplatelet therapy in atrial fibrillation: Review of the literature and future avenues. J Cardiovasc Electrophysiol 2003; 14 (Suppl): S60-S63.

34. Blackshear J, Baker V, Rubino F. Adjusted-dose warfarin versus low-intensity, fixed-dose warfarin plus aspirin for high-risk patients with atrial fibrillation-stroke prevention in atrial fibrillation III randomized clinical trial. Lancet 1996; 348: 633-8.

35. Pérez-Gómez F, Alegría E, Berjón J, Iriarte J, Zumalde J, Salvador A et al. For the naspeaf investigators. Comparative Effects of Antiplatelet, Anticoagulant, or Combined Therapy in Patients With Valvular and Nonvalvular Atrial Fibrillation A Randomized Multicenter Study. J Am Coll Cardiol 2004; 44: 1557-66.

36. Stoddard M, Dawkins P, Prince C, Ammash N. Left atrial appendage thrombus is not uncommon in patients with acute atrial fibrillation and a recent embolic event: a transesophageal echocardiographic study. J Am Coll Cardiol 1995; 25: 452-9.

37. Blackshear J, Johnson D, Odel J, Baker V, Howard M, Pearce L et al. Thoracoscopic Extracardiac Obliteration of the Left Atrial Appendage for Stroke Risk Reduction in Atrial Fibrillation. J Am Coll Cardiol 2003; 42: 1249-52.

38. Katz E, Tsiamtsiouris T, Applebaum R, Schwartzbard A, Tunick P, Kronzon I. Surgical left atrial appendage ligation is frequently incomplete: a transesophageal echocardiographic study. J Am Coll Cardiol 2000; 36: 468-71.

39. Sievert H, Lesh M, Trepels T, Ogi D, Goldman L, SCHERER D. Percutaneous Left Atrial Appendage Transcatheter Occlusion (PLAATO) to Prevent Stroke in Patients With Atrial Fibrillation: First Human Experience. J Am Coll Cardiol 2002; 39 (Suppl 1): 6A-7A.

40. SieverT H, Lesh MD, Trepels T. Percutaneous left atrial appendage transcatheter occlusion to prevent stroke in high-risk patients with atrial fibrillation- early clinical experience. Circulation 2002; 105: 1887-9.

41. Hanna I, Kolm P, Martin R, Reisman M, Gray W, Bцоск P. Left atrial structure and function after percutaneous left atrial appendage transcatheter occlusion (PLAATO): six-month echocardiographic follow-up. J Am Coll Cardiol 2004; 43: 1868-72.

42. Ostermayer S, Reisman M, Kramer P, Matthews R, Gray W, Block P et al. Percutaneous Left Atrial Appendage Transcatheter Occlusion (PLAATO System) to Prevent Stroke in High-Risk Patients With Non-Rheumatic Atrial Fibrillation. Results From the International Multi-Center Feasibility Trials. J Am Coll Cardiol 2005; 46: 9-14. 